\title{
POLYARTHRITIS AND VAGINAL MELANOMA
}

Maria Clara Cardoso Gomes Zampirolli1,*, Clarice Garcia Valadares Xavier ${ }^{1}$, Samara de Quadros Lobê ${ }^{1}$, Aurivan Essado Dantas ${ }^{1}$, Matheus Fonseca Cardoso ${ }^{1}$, Maria Raquel Costa Pinto ${ }^{1}$, Maria Fernanda Brandão de Resende Guimarães ${ }^{1}$

1.Universidade Federal de Minas Gerais, Belo Horizonte (MG), Brazil.

*Corresponding author: mariaclarazamp@gmail.com

\section{BACKGROUND}

Dysfunctional immune system as a result of underlying malignancy can trigger a universe of signs and symptoms unrelated to tumor or to metastasis. This condition, named paraneoplastic syndrome (PS), rare in rheumatology, manifests with hypertrophic osteoarthropathy, paraneoplastic polyarthritis, paraneoplastic vasculitis, tumor-induced osteomalacia and cancer-associated myositis. To diagnose PS, temporal and causal criteria must be considered according to the Bradford Hill Criteria and the symptoms must appear simultaneously or may precede the appearance of cancer in up to 2 years. The best evidence of causality is established when there is resolution of rheumatic manifestations with the therapeutic success of cancer.

\section{CASE REPORT}

A 50-year-old woman presented at an arthritis clinic with symmetrical and additive polyarthritis of hands, knees and elbows for the last 2 months, and weight loss (13 kg in the last 8 months). Her comorbidities were arterial hypertension, diabetes mellitus and smoking. Rheumatoid factor (RF) and anticitrullinated protein antibodies (ACPA) were present in high titers, antinuclear antibody was 1:80 (nuclear dense fine speckled pattern) and erythrocyte sedimentation rate was prolonged. Serology for HIV, B and C hepatitis were negative. A vaginal biopsy was performed two months earlier to investigate blackened vaginal discharge. Nodular melanoma with vascular invasion (Breslow depth of $17 \mathrm{~mm}$ ) was evidenced. Partial resection of the vaginal lesion was performed, followed by radiotherapy and chemotherapy (CT). The treatment was performed with injectable and oral corticosteroids (CS) and subsequently with methotrexate ( $20 \mathrm{mg} /$ week), with partial response to treatment. Eight months after the diagnosis of cancer, she had sudden right hemiparesis with brain image evidencing metastatic disease and she evolved to death.

\section{CONCLUSION}

Paraneoplastic syndrome may worsen quality of life, morbidity and mortality of malignancy. Cancer screening should be guided by the clinical history and appropriate recommendations for age and sex. Although, increased awareness of rheumatic symptoms related to malignant diseases may enable early neoplasia diagnosis. Poor response to CS and disease-modifying antirheumatic drugs should be a clinical warning for PS, since there are no biomarkers to help distinguish it from other rheumatic conditions yet. Description of rheumatoid arthritis-like syndrome in patient with malignancy emphasizes the importance of awareness of PS as a mimic of rheumatic diseases. 